\title{
I - INTERNATIONAL PUBLICATIONS OF THE FACULTY - ARTICLES AND BOOKS (2000-2003)
}




\section{ARGENTINA}

1 - 2000 - AGUIAR JÚNIOR, Ruy Rosado de. Daños Derivados de Actos Del Poder Judicial. In: Los Nuevos Daños: soluciones modernas de reparación. vol. 1, 2 ed. renov. amp., Carlos Alberto Ghersi (Coordinador). Buenos Aires: Hammurabi, 2000, p. $277-320$.

2 - 2000 - JAEGER JUNIOR, Augusto. Los indios, los jesuitas y el Mercosur. Revista de Derecho del Mercosur. Buenos Aires: La Ley, a. 4, n. 3, jun. 2000. p. 19-24. (Dr. candidate PPGDIR./UFRGS)

3-2000-MARTINS-COSTA, Judith. Direito e Cultura: entre as veredas da existência e da história. Investigación y Docência. n. 33, Rosário, Argentina: Universidade Nacional de Rosário - Fundación para lãs Investigaciones Jurídicas, p.91-103. (waiting confirmation)

4- 2000 - LIMA MARQUES, Claudia. Contratos de Time-Sharing en Brasil y la Protección de los Consumidores: critica al derecho civil en tiempos postmodernos. In: Los Nuevos Daños: soluciones modernas de reparación. vol. 2. Carlos Alberto Ghersi (Cootdinador), Buenos Aires: Hammutabi, 2000, p. 69-105.

5-2000 - LIMA MARQUES, Claudia. TURKIENICZ, Eduardo. En defensa de la teoría finalista para la interpretación del artículo $2^{\circ}$ del Código de Defensa del Consumidor Brasileño. Revista de Responsabilidad Civily Seguros La Ley. Buenos Aires, v. Vl, 2000, p. 16-29. (waiting confirmation)

6- 2000 - OLIVAR JIMENEZ, Martha Lucia. Establecimiento de una Politica Comun de Proteccion al Medio Ambiente. Su Necessidad dentro de um Mercado Comun. In: Nuevos Daños: soluciones modernas de reparación. vol. 1. Carlos Aberto Ghersi (Coordinador). Buenos Aires: Hammurabi, 2000, p. 229-264. (waiting confirmation)

7 - 2001 - MARTINS-COSTA, Judith Hofmeister. Los Daños a la Persona en el Derecho Brasileño y la Naturaleza de su reparación. Revista de Responsabilidade Civil y Seguros - La Ley. año 3, n. 2. Buenos Aires, mar./abril - 2002. (waiting confirmation)

8 - 2001 - LIMA MARQUES, Claudia. Algunas observaciones sobre la protección del consumidor y del usuario de baja renta en los servicios bancarios brasileños. In: Obligaciones y contratos en los albores del siglo XXI: Homenagem ao Professor Dr. Roberto López Cabana. Oscar J. Ameal and Silvia Y. Tanzi (Org). Buenos Aires: Abeledo-Perrot, 2001, p. 797-811. (waiting confirmation) 
9 - 2001 -LIMA MARQUES, Claudia. A Insuficiente Proteção do Consumidor nas Normas de Direito Internacional Privado: da necessidade de uma Convenção Interameticana (CIDIP) sobre a lei aplicável a alguns contratos e relações de consumo. Revista de Derecho Internacional y dela Integración. Santa Fé, 2001. (waiting confirmation)

10-2002-DEL'OLMO. Florisbal de Souza. Augusto Teixeira de Freitas: el protojurista del Mercosur. Buenos Aires: Lecciones y Einsayos, 2002.(Dr. candidate PPGDIR./UFRGS)

11 - 2003-ALVARO DE OLIVEJRA, Carlos Alberto. O processo civil na perspectiva dos direitos fundamentais. Revista Iberoamericana de Derecho Procesal, 2002, III, p. 45-60.

12 - 2003 - JAEGER JUNIOR, Augusto. Prólogo. In: TANSISI, Nilda. Administración de Marketing: Segmentación integral de mercados. Posadas: Graficop, 2003. p. 14-17. (Dt. candidate PPGDIR./UFRGS)

13 - 2003 - MARTINS-COSTA, Judith Hofmeister. Buena Fé y Adimplemento das Obligaciones. In: CORDOBA, Marcos; GARRIDO, Lídia; KLUGER, Viviana. (Org). Tratado de la Buena Fé. Buenos Aires, 2003. (waiting confirmation)

1.4 -2003 - LIMA MARQUES, Claudia. O Direito do Mercosul: Direito Oriundo do Mercosul, entre direito internacional clássico e novos caminhos de integração. Derecho Del Mercosur y la Integración, n. 1, feb, 2003, p. 61-89. (wating confirmation)

15 - 2003-LIMA MARQUES, Claudia. Cap. 6 - Derecho aplicable - V. Técnicas de reglamentación directas. In: Derecho Internacional Privado de los Estados del Mercosur. Diego P. Fernandez Arroyo (Org.), Buenos Aires: Zavalia, 20003, p. 272-277. (waiting confirmation)

16-2003-LIMA MARQUES, Claudia. FERNÁNDEZ-ARROYO, Diego P. (Spain), Cap. 15 - Protección de menores en general. In: Derecho Internacional Privado de los Estados del Mercosur. Diego P. Fernandez Arroyo (Org), Buenos Aires: Zavalia, 20003, p. 584-594. (waiting confirmation)

17 - 2003 - LIMA MARQUES, Claudia. FRESNEDO AGUIRRE, Ceclia. (Uruguay) Cap. 14- Personas juridicas. In: Derecho Internacional Privado de los Estados del Mercosur. Diego P. Fernandez Arroyo (Org), Buenos Aires: Zavalia, 20003, p. 541-554. (waiting confirmation)

18 - 2003 - LIMA MARQUES, Claudia. Cap. 17 - Filiación. OPPERTI, Didier. (Utuguay) In: Derecho Intemacional Privado de los Estados del Mercosur. Diego P. Fernandez Arroyo (Org), Buenos Aires: Zavalia, 20003, p. 647-656. (waiting confirmation)

19 - 2003 - LIMA NARQUES, Claudia; LORENZET'T'T, Ricardo Luis. (Argentina) Teoria General de los Servicios de Consumo. Vol. 1. Buenos Aires: Rubinzal-Culzoni, 2003/2004.140p. (wating confirmation) 


\section{BELGIUM}

1 -2000-JACOB DE FRADERA, Véra Maria. Cap. Langue et Droit au Mercosur. Langue et Droit. (Erik Jayme -org.) Bruxells: Bruylant, 2000, p. 123-140. (waiting confirmation)

2 - 2000-JAEGER JUNIOR, Augusto. Mercosul e a liberdade de concorrência. In: Boletín Latinoamericano de Competência. Bruxelas, dez. 2000, n. 11, p. 25-31.(Dt. candidate PPGDIR./UFRGS)

3 - 2000 - LIMA MARQUES, Claudia; STIGLJTZ, Gabriel (Argentina). Uruguay: Consumer Protection Act Adopted. Consumer Law Journal. Belgium, v. 8, n. 1, 2000 , p. 80. (waiting confirmation)

4- 2000 - LIMA MARQUES, Claudia. The Cigarette Industry has the burden of providing the nicotine is not addictive to consumers. Consumer Law Journal. Betgium, v. 8, n. 2, 2000, p. 204-205. (waiting confirmation)

5 - 2001 - JAEGER JUNIOR, Augusto. Para uma quinta liberdade econômica fundamental. In: Boletín Latinoamericano de Competência. Bruxelas, nov. 2001, n. 13, p. 38-49. (Dr. candidate PPGDIR./UFRGS).

6-2001 - GUIMARÃES TABORDA, Maren. La Jurisprudence classique romaine et la construction d'un droit des affaires fondé sur la fides. Revue Internationale des Droits de L'Antiquité. Bruxelles: , v.XLVIII, n.1, p.151 - 183, 2001

7 . 2002 - JAEGER JUNIOR, Augusto. O novo Código Civil Brasileiro e a reorganização de cmpresas. In: Boletín Latinoamericano de Competência. Bruxelas, oct. 2002, n. 15, p. 53-77. (Dt. candidate PPGDIR./UFRGS) 


\section{BOLIVIA}

1 - 2000 - DEL'OLMO, Florisbal de Souza. A Naturalização nos Estados HispanoAmericanos do Mercosul. In: IX Encuentro Internacional de Derecho de América do Sur. Los Procesos de Integración en el Nuevo Milénio. La Paz: Universidad Católica Boliviana, 2000, p. 157-161. (Dr. candidate PPGDIR./UFRGS).

2-2000 - JAEGER JUNIOR, Augusto. Mercosul e a liberdade de concorrência. In: PIMENTEL, Luiz Otávio; NOGALES DE SANTIVÁÑEZ, Emma. (Orgs.) IX Encuentro Internacional de Derecho de América del Sur: los procesos de integración en el nuevo milenio. La Paz: Universidad Católica Boliviana, 2000. p. 17-24. (Dr. candidate PPGDIR./UFRGS). (waiting confirmation) 


\section{COLOMBIA}

2002- ALVARO DE OLIVEIRA, Carlos Alberto. Livre apreciação da prova: perspectivas batuais. In: Prueba - Homenaje al maestro Hernando Devis Echandía. Colombia: Universidad Libre, 2002, p. 599-618. 


\section{FRANCE}

1 - 2002 - JACOB DE FRADERA, Véra Maria. Réflexions sur l'Apport du Droit Comparéa l'Élaboration du Droit Communautaire, 2002. Dr. Thesis, Université de Paris I, mention très honorable et les felicitations du jury. (waiting confirmation)

2 -2003 - JACOB DE FRADERA, Véra Maria. La responsabilité. In: Aspects Nouveaux, Travaux de l'Association Henri Capitant. Journées Panaméennes, tome L/ 1999, p. 217-220. Paris: LGDJ, 2003, p. 217-220.

3 - 2003 - JACOB DE FRADERA, Véra Maria. Le rôle du droit comparé dans l'avènement du droit européen. The Role of Comparative Law in the Emergence of European Law. In: Revue Internationale de Droit Comparé, n. 2, 2003, p. 484-486. 


\section{GERMANY}

1 - 2000- WEHNER, Ulrich, EU und Mercosur -Auf dem Weg zur Freihandelszone? (União Européia e Mercosul: No caminho de uma zona de livre comércio ?"). Recht der Internationalen Wirtschaft, ano 2000, p. 370 a 376. (DAAD/CAPES, permanent lector, PPGDir./UFRGS, Germany). (waiting confirmation)

2 - 2001 - HECK, Luís Afonso. Die Normenkontrolle im brasilianischen Verfassungstecht. In: GEIS, Max-Emanuel; LORENZ, Dieter (Org). Staat-Kirche-Verwealtung: Festschrift für Hartmut Maurer zum 70. Geburstag. Vol. 1. München, 2001, p. 111-126.

3- 2002 - ÁVILA, Humberto Bergmann. Materiell verfassungsrechtliche Beschraenkungen der Besteuerungsgewalt. Baden Baden: Nomos-Verlag-Gesellsach ft, 2002, 398p. (waiting confirmation)

4 - 2002 - LIMA MARQUES, Claudia. Gleichheit zwischen Kindern im aktuelle brasilianischen Recht- Postmodernes Recht? Rechtsentwicklungen in Portugal, Brasilien und Macau. Erik Jayme and Christian Schindler (Hrsg), Nomos Verlagsgesellschaft, BadenBaden, 2002, p. 65-98. (waiting confirmation)

5-2002-MICHELON JUNIOR, Claudio Fortunato. The Justification of Authority and the Insularion of Formal Reasons. Archiv für Rechts und Sozialphilosophie (ARSP). Vol. 88, n. 1. Bielefeld, 2002, p. 51-73.

6-2003-LIMA MARQUES, Claudia. Das neue brasilianische Zivilgesetzbuch von 2002: Bemerkungen zum neuen Unternehmensrecht (Direito de Empresa) und der Quellendialog mit dem Verbraucherschutzgesetzbuch von 1990. In: Das Recht der lusophonen Länder II. Erik Jayme and Christhoph Benicke (Hrsg), Nomos Verlagsgesellschaft, BadenBaden, 2003, DLJV Erlangen, 21 pg. 


\section{GREECE}

2003- LIMA MARQUES,Cláudia. OGM et le droit de la consommation: une action mondial pour assurer l'information des consommateurs? In: 9th Internation Consumer Law Conference. Athens, Greece, 2003 (CD). 


\section{ITALY}

1 - 2000 - MARTINS-COSTA, Judith Hofmeister. Os Danos à Pessoa no Direito Brasileiro e a Natureza da Repatação. Revista Roma e América - Diritto Romano Comune, n. 10, separata, Roma: Mucchi Editore, 2000, p. 157-185. (waiting confirmation)

2 - 2002 - MARTINS-COSTA, Judith Hofmeister. A Boa-fé como Modelo. Revista Roma e América - Diritto Romano Comune, n. 13, separata, Roma: Mucchi Editore, 2002, p. $71-98$.

3-2002-LIMA MARQUES, Claudia. Défis d'un dialogue entre les règles régionales, universelles er du Mercosur en matière de procédure civile internationale. In : Congress to Celebrate the 75th Anniversary of the Fouding of the International Institute for the Unification of Private Law (UNIDROIT). Harmonisation Mondiale du Droit Privé et Integration économique régionale. Rome, 2002 (CD). (waiting confirmation)

4-2003-LIMA MARQUES, Claudia. Procédure civile internationale et Metcosur : pour un dialogue des règles universelles et régionales. Revue du Droit Uniforme/ Uniform Law Review - UNIDROIT, vol. VIII, 2003-1/2, Roma, p. 465-484. (waiting confirmation)

5- 2003 - ALVARO DE OLIVEIRA, Carlos Alberto. Mauro Cappeletti and the Brazilian Procedural Law. International Association of Ptocedutal Law (Ed.), In honor Mauro Capeletti, Milan, CEDAM, 2003/2004.

6- 2003 - ALVARO DE OLIVEIRA, Carlos Alberto. Considerazioni intorno all'eficacia della sentenza. International Association of Procedural Law (Ed.), In bonor Giuseppe Tarzia, Milan, CEDAM, 2003/2004. 


\section{PERU}

1 - 2002-ALVARO DE OLIVEIRA, Carlos Alberto. A Garantia do Contraditório. Revista Peruana de Derecho Procesal, vol. V, junho/2002, p. 11-23. (waiting confirmation)

2 - 2003- AlVARO DE OlVEIRA, Carlos Alberto. Efetividade e Processo de Conbecimento. Revista Peruana de Derecho Procesal, vol. VI, maio/2003, p. 13-26.

3-2003-ROJO, Raúl Enrique. De revoluciones y reformas: Tras un tipo ideal de la reforma social. In: $X X I V^{\circ}$ Congreso de la Asociación Latinoamericana de Sociologia. Arequipa, Peru: Universidad Nacional de San Agustín de Arequipa (CD-ROM América Latina: bacia una nueva Alternativa de desarrollo). 


\section{PORTUGAL}

1 - 2002 - MARTINS-COSTA, Judith Hofmeister. Culturalismo e Experiência no Novo Código Civil. Boletim da Faculdade de Direito de Coimbra, v. XXVIII, Coimbra, 2002, p. 603-627.

2 - 2002 - LIMA MARQUES, Claudia. A Convenção de Haia de 1993 e o regime da adoção internacional no Brasil após a aprovação do novo Código Civil Brasileiro em 2002. In: Estudos em Homenagem à Professora Doutora Isabel Magalhães Collaço, vol. I. Universidade de Coimbra: Almedina, Portugal, p. 263-309.

3 - 2002- AZEVEDO DA SILVEIRA, Patrícia. AZEVEDO MARQUES, Vicente. Por quem os sinos dobram: o leading case das queimadas no RS. Revista de Ciência e Cultura, Série Direito, Universidade Lusíada-Porto, n. 1 e 2, ano 2002, p. 569-584.(Dr. candidate PPGDIR./UFRGS) 


\section{SPAIN}

2003 - LIMA MARQUES, Claudia. O Direito Internacional privado solucionando 'conflitos de cultura': os divórcios no Japão e seu reconhecimento no Brasil. In: El Derecho de Família ante el siglo XXI: Aspectos internacionales. Alfonso-Luis Calvo Caravaca e Esperanza Castellanos Ruiz (Direc.). Madrid: Colex, 2003/2004, p. 547-572. 


\section{UNITED STATES OF AMERICA}

1 - 2000 - LIMA MARQUES, Claudia. Assisted Reproduction Technology in South America and the Effect on Adoption. Texas International Law Journal. Vol. 35, n. 1, 2000. p. 65-91. (waiting confirmation)

2- 2001 - LIMA MARQUES, Claudia. Banking in the information society: a Brazilian vision. Consumer Law in the Information Society. Thomas Wilhelsonn, Salla Tuominen and Heli Tuomola (Ed.), Kluwer Law International, Cambridge (USA), The Hague/ London/Boston, 2001, p. 247-262.

3 - 2001 - LIMA MARQUES, Claudia. A proteção do consumidor: aspectos de direito privado regional e geral. XXVII Curso de Derecho Internacional -OEA/CIJ. Washington: Secretaría General - Subsecretaria de Asuntos Jurídicos, 2001, p. 657-780. (waiting confirmation)

4-2001 -MARTINS-COSTA, Judith Hofmeister. O Projeto de Código Civil Brasileiro - em Busca da Ética da Situação. Revista Jurídica de la Universidad Interamericana de Puerto Rico. v. 35, n. 3, mayo-ago, 2001, p. 443-428.

5 - 2002 - LIMA MARQUES, Claudia. A proteção do consumidor: aspectos de direito privado regional e geral. In: El Derecho Internacional Privado en las Américas (1974-2000), Cursos de Derecho Internacional - vol. I (Parte 1). Washington: Secretaría General - Subsectetaria de Asuntos Jurídicos, 2002, p. 1503-1622. (waiting confirmation)

6-2003-JACOB DE FRADERA, Véra Maria. Report on Constitution, International Treaties and Contracrs. The $16^{\text {th }}$ International Congress of Comparative Law. Huntington, NY: Managing Legal Editor Juris, 2003. 